\title{
Lunar Lander Landing Site Decision in Low-Fuel Case
}

\author{
Min-Guk Seo, Seong-Min Hong and Min-Jea Tahk \\ KAIST, Department of Aerospace Engineering, Daejeon, Korea
}

\begin{abstract}
This paper deals with lunar landing site decision algorithms for the case when the lunar lander is not able to reach the original landing site. A new landing site which minimizes the fuel consumption of the lunar rover is determined from landing site candidates located within the maximum reachable downrange of the lunar lander. A path planning algorithm is introduced to determine the new landing site which has the shortest path to the original landing site among the candidates. Numerical simulations are conducted to verify the performance of the proposed algorithm.
\end{abstract}

\section{Introduction}

In a lunar landing mission, the lander may need a new landing site if the landing on the original landing site is not possible due to various disturbances during the operation. A good candidate for the new landing site is an area from which the rover is able to travel to the original landing site, where the mission should take place, with minimum energy consumption. A new landing site decision algorithm is designed with full information of obstacles to satisfy this requirement. The first step of the algorithm is to obtain the safe reachable region of a lunar lander by solving the landing trajectory optimization problem for maximum downrange. The new landing site is chosen among all the landing site candidates as the one whose path to the original landing site is determined to be the shortest one by applying path planning algorithm.

Landing guidance is an important issue in the planetary exploration field. The common objective of planetary landing guidance algorithms of previous studies is to precisely land on the predetermined landing site with minimum fuel consumption[1-5]. This implies that those algorithms are not suitable to figure out the maximum downrange of the lunar lander under fuel limits. In this paper, the optimal landing guidance problem is reformulated as a downrange maximization problem. The dynamics of the lunar lander is simply modeled in a 2dimensional local Cartesian coordinate system. The optimal solution is obtained using Gauss Pseudospectral Optimization Solver (GPOPS). The outer-most contour points of the reachable landing region, which are new landing site candidates, are determined from the maximum reachable downrange and the obstacle information.

Path planning algorithms with obstacle avoidance studied in many previous articles[6-13] do not focus on guaranteeing shortest path. This paper suggests a new path planning algorithm especially for the lunar rover to minimize path length. The obstacles on the lunar surface are assumed to be given as circles and polygons. The resulting path map consists of straight line segments and circular arcs. The shortest path starting from the landing site is obtained by applying Dijkstra's algorithm[14]. The landing site candidate with the minimum path length is chosen as the new landing site.

This paper is organized as follows. The landing trajectory optimization for downrange maximization is handled in Section 2. The path planning algorithm for lunar lander is introduced in Section 3. Simulation results are given in Section 4 for the performance analysis of the algorithm. The overall conclusion of this paper is addressed in Section 5.

\section{Landing Trajectory Optimization for Downrange Maximization}

In the case when a lunar lander cannot reach the original landing site where exploration missions are supposed to be performed, a landing site decision algorithm is needed to find a new landing site. One requirement of the new landing site is that the lunar lander should be able to land on it safely with limited amount of fuel of the thruster and the attitude control system. This implies that the landing site candidates should be the points where the lunar lander can make a landing and not on obstacle regions. The lunar rover has to travel to the original landing site to perform its missions after the lunar lander reaches the new landing site. In order to minimize the energy and the time consumption, the point which has the shortest path to the original landing site is chosen as the new landing site among candidate points.

As mentioned previously, the points in the reachable region for lunar lander on the lunar surface are candidates of the new landing site. Since the original landing site is 
supposed to be outside of the reachable region in this paper, all the paths from the candidates should cross the outer-most contour of the reachable landing region. This means that the landing site candidates are limited to the points on the outer-most contour. The landing trajectory optimization for downrange maximization is utilized to figure out the contour.

Several assumptions are considered to define landing trajectory optimization problem.

1) The motion of the lunar lander is described in inertial Cartesian coordinate system with its origin on the lunar surface, which is assumed to be a flat plane.

2) The altitude axis is normal to the lunar surface and the other axes are on the surface.

3) The effect due to the rotation of the moon is neglected and the uniform gravitational acceleration is considered.

4) The initial velocity component normal to the altitude axis and the attitude angle rate are 0 .

5) The major axis of the lunar lander, the axis parallel to the main thruster direction, is parallel to the altitude axis at the initial time.

6) The rotation with respect to any axis normal to the major axis is possible by the combination of the attitude control system.

Under those assumptions, 1) 6), the trajectory optimization problem is defined in the 2-dimensional inertial Cartesian coordinate system, and the outer-most contour is defined as a circle centered at the original landing site with the maximum downrange as the radius. The effect of altitude control thrusters on the lunar lander is velocity change, and the attitude control is conducted by attitude control system equipped on the lander. The control inputs of the lunar lander are defined as main thruster and attitude thruster in this model The dynamic model of the lunar lander is defined from all the assumptions given above.

$$
\begin{aligned}
& \dot{x}=V_{x} \\
& \dot{h}=V_{h} \\
& \dot{V}_{x}=\frac{T_{D} \cos \theta}{m} \\
& \dot{V}_{h}=\frac{T_{D} \sin \theta}{m}-g \\
& \dot{m}=-\frac{\left(T_{D}+T_{a t t}\right)}{I_{s p} g} \\
& \dot{\theta}=q \\
& \dot{q}=\frac{T_{a t t} \cdot L_{T}}{I_{z z}}
\end{aligned}
$$

where $x$ and $h$ are downrange and altitude of lunar lander. $V_{x}$ and $V_{h}$ denote velocity components in each axis direction. Mass, moment of inertia, moment arm of the attitude thruster, attitude angle, and attitude angle rate of lunar lander are symbolized as $m, I_{z z}, L_{T}, \theta$, and $q$. The attitude angle is defined as the angle between the major axis and the downrange axis. $I_{s p}$ is specific impulse of lunar lander engine, which is used at the main thruster and attitude control thruster, and $g$ is the gravitational acceleration on the lunar surface. The control input variables are main thrust, $T_{D}$, and attitude control thrust, $T_{a t t}$.

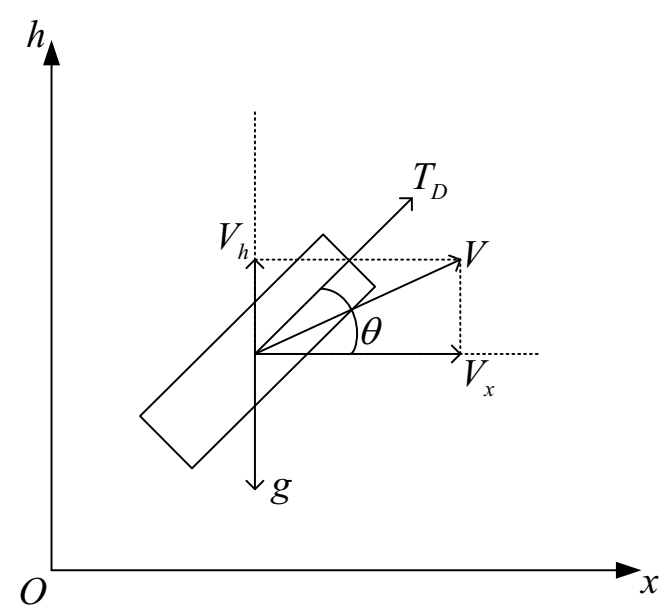

Figure 1. Coordinate System and State Variable Definitions.

The objective function is defined to maximize the magnitude of $x$.

$$
\min _{T_{D}, T_{\text {att }}}-|x|
$$

The initial conditions of state variables are given from the assumptions addressed before.

$$
\begin{array}{lccc}
x\left(t_{0}\right)=x_{0} & h\left(t_{0}\right)=h_{0} & V_{x}\left(t_{0}\right)=0 & V_{h}\left(t_{0}\right)=0 \\
m\left(t_{0}\right)=m_{0} & \theta\left(t_{0}\right)=90^{\circ} & q\left(t_{0}\right)=0
\end{array}
$$

The altitude of the lunar lander is bounded to be larger than 0 to protect it from crashing on the lunar surface. The sensors like cameras on the lunar lander measure the information of the lunar surface for safe landing and hazard avoidance. The attitude angle and its rate are limited to consider the operation performance of those sensors.

$$
\begin{array}{ccc}
h\left(t_{f}\right)=0 & V_{x}\left(t_{f}\right)=0 & V_{h}\left(t_{f}\right)=0 \\
\theta\left(t_{f}\right)=90^{\circ} & q\left(t_{f}\right)=0 \\
h \geq 0 & \\
\theta_{\min } \leq \theta \leq \theta_{\max } & \\
q_{\min } & \leq q \leq q_{\max }
\end{array}
$$

The main thrust is limited by the performance of the actuator. The attitude control thruster is also limited under actuator performance, but it can be a negative value considering both direction of the thruster.

$$
\begin{aligned}
& T_{D_{\min }}<T_{D}<T_{D_{\max }} \\
& T_{a t t_{\min }}<T_{a t t}<T_{a t_{\max }}
\end{aligned}
$$

The restriction on the amount of fuel is expressed as a mass limit of the lunar lander.

$$
m_{\min } \leq m \leq m_{\max }
$$

The solution of the optimization problem defined in (1)-(7) is obtained by applying a proper optimization algorithms. GPOPS is the optimization tool utilized in this paper. This tool is based on the variable-order Gaussian quadrature method and deals with continuoustime optimal control problems. The outer-most contour of the reachable region is decided from the optimal solution 
as the circle on the lunar surface centered at the initial position on the surface with the maximum downrange as radius. Assuming that the information of obstacles are given, the landing site candidates are defined as the points dividing this contour into certain small and equal intervals and not located on the obstacles.

\section{Path Planning Algorithm for Lunar Rover}

As addressed before, the new landing site is the one among candidates with the minimum path length to the original landing site. The path planning algorithm between two points is required for this process. The designed algorithm should not only ensure the minimum path length but also guarantee the safety of lander by planning the path not to cross the obstacles on the lunar surface.

The path planning algorithm introduced in this paper starts from constructing path candidates from a landing site candidate to the original landing site. The obstacles on the lunar surface are assumed to be given as geometrical figures as stated below. Each of the original landing site and a landing site candidate is able to be expressed as a point on the lunar surface. The craters are able to be approximated as circles[15]. The circular obstacle centered at $\left(x_{c}, y_{c}\right)$ with the radius of $r_{c}$ is expressed as $C\left(x_{c}, y_{c}, r_{c}\right)$ in this paper. The information of other obstacles which are not round, like rocks, are able to be detected from the sensor measurements[16]. Each of those obstacles is able to be approximated as a polygon, which is figure consists of straight segments, surrounding it[17].

In the case when the obstacles are circles, the minimum path is consists of some of following path segment candidates: tangent lines from two end points to the circles, common tangent lines of two arbitrary selected circles, and circular arc connecting points of contact on each circle[18]. When the obstacles are polygons, path segment candidates are as follows: straight segments between two end points and the vertexes of the polygons, straight segments between two vertexes from different polygons, and the sides of the polygons[19]. Since the obstacles on the lunar surface includes both circles and polygons, the group of path segment candidates consists of all the sorts of them stated above and the tangent lines from the vertexes of the polygons to the circular obstacles.

The straight line connecting two points, $\left(x_{1}, y_{1}\right)$ and $\left(x_{2}, y_{2}\right)$, are given as follows.

where

$$
a_{P P} x+b_{P P} y+c_{P P}=0
$$

$$
\begin{aligned}
& a_{p p}=y_{2}-y_{1} \\
& b_{p p}=-\left(x_{2}-x_{1}\right) \\
& c_{p p}=-x_{1}\left(y_{2}-y_{1}\right)+y_{1}\left(x_{2}-x_{1}\right)
\end{aligned}
$$

The path segment is defined by (8) and two end points, $\left(x_{1}, y_{1}\right)$ and $\left(x_{2}, y_{2}\right)$.
The two tangent lines to the circle $C\left(x_{c}, y_{c}, r_{c}\right)$ from the point $\left(x_{p}, y_{p}\right)$ outside of the circle are obtained as below.

$$
a_{C P} x-y+c_{C P}=0
$$

where

$$
\begin{aligned}
a_{C P} & =\frac{f_{C P} \pm \sqrt{f_{C P}^{2}-g_{C P}}}{\left(x_{c}-x_{p}\right)^{2}-r_{c}^{2}} \\
c_{C P} & =y_{p}-a x_{p} \\
f_{C P} & =\left(x_{c}-x_{p}\right)\left(y_{c}-y_{p}\right) \\
g_{C P} & =\left[\left(x_{c}-x_{p}\right)^{2}-r_{c}^{2}\right]\left[\left(y_{c}-y_{p}\right)^{2}-r_{c}^{2}\right]
\end{aligned}
$$

The points of contact on the tangent lines, $\left(x_{t}, y_{t}\right)$, obtained as (10) and (11) are calculated as,

where

$$
\begin{aligned}
& x_{t}=\frac{f_{x_{t}} \pm \sqrt{f_{x_{t}}^{2}-g_{x_{t}}}}{a_{C P}^{2}+1} \\
& y_{t}=a_{C P} x_{t}+c_{C P}
\end{aligned}
$$

$$
\begin{aligned}
& f_{x_{t}}=\left[a_{C P}\left(c_{C P}-y_{c}\right)-x_{c}\right] \\
& g_{x_{t}}=\left(a_{C P}^{2}+1\right)\left[x_{c}^{2}+\left(c_{C P}-y_{c}\right)^{2}-r_{c}^{2}\right]
\end{aligned}
$$

The equations of path segment candidates are given as (10), and the end points of them are $\left(x_{p}, y_{p}\right)$ and $\left(x_{t}, y_{t}\right)$.

The common tangent lines between two circles, $C\left(x_{c_{1}}, y_{c_{1}}, r_{c_{1}}\right)$ and $C\left(x_{c_{2}}, y_{c_{2}}, r_{c_{2}}\right)$, are given as follows.

where

$$
a_{C C} x+b_{C C} y+c_{C C}=0
$$

$$
\begin{gathered}
a_{C C}=\frac{f_{x_{c}} f_{r_{c}}+f_{y_{c}} \sqrt{f_{x_{c}}^{2}+f_{y_{c}}^{2}-f_{r_{c}}^{2}}}{f_{x_{c}}^{2}+f_{y_{c}}^{2}} \\
b_{C C}=\frac{f_{y_{c}} f_{r_{c}}-f_{x_{c}} \sqrt{f_{x_{c}}^{2}+f_{y_{c}}^{2}-f_{r_{c}}^{2}}}{f_{x_{c}}^{2}+f_{y_{c}}^{2}} \\
c_{C C}=-\left(x_{c_{2}} a_{C C}+y_{c_{2}} b_{C C} \pm r_{c_{2}}\right) \\
f_{x_{c}=\left(x_{c_{1}}-x_{c_{2}}\right) \quad f_{y_{c}}=\left(y_{c_{1}}-y_{c_{2}}\right) \quad f_{r_{c}}}=\left( \pm r_{c_{1}} \pm r_{c_{2}}\right)
\end{gathered}
$$

The point of contact on $C\left(x_{c_{1}}, y_{c_{1}}, r_{c_{1}}\right),\left(x_{t_{1}}, y_{t_{1}}\right)$, and one on $C\left(x_{c_{2}}, y_{c_{2}}, r_{c_{2}}\right),\left(x_{t_{2}}, y_{t_{2}}\right)$, are calculated as,

$$
\begin{array}{ll}
x_{t_{1}}=x_{c_{1}}-\left( \pm r_{c_{1}} a_{C C}\right) & y_{t_{1}}=y_{c_{1}}-\left( \pm r_{c_{1}} a_{C C}\right) \\
x_{t_{2}}=x_{c_{2}}+\left( \pm r_{c_{2}} a_{C C}\right) & y_{t_{2}}=x_{c_{2}}+\left( \pm r_{c_{2}} a_{C C}\right)
\end{array}
$$

The mathematical signs in front of the same variable are equal. This results in four common tangent lines except for $\left(x_{c_{1}}-x_{c_{2}}\right)^{2}+\left(y_{c_{1}}-y_{c_{2}}\right)^{2}<\left(r_{c_{1}}+r_{c_{2}}\right)^{2}$ case. Two tangent lines, obtained when the mathematical signs in front of $r_{c_{1}}$ and $r_{c_{2}}$ of (15) are different from each other, and the points of contact on them are possible in this case. The equations of path segment candidates are 
expressed as (14). $\left(x_{t_{1}}, y_{t_{1}}\right)$ and $\left(x_{t_{2}}, y_{t_{2}}\right)$ are the end points of them.

The path candidates should be designed not to pass through any of obstacles for safety of the lunar rover. This implies that the path segment candidates, whose part is included in any of the obstacles, should be excluded. The acceptability test is designed for an arbitrary path segment candidate, denoted by two end points, $\left(x_{1}, y_{1}\right)$ and $\left(x_{2}, y_{2}\right)$, and the equation of straight line, $a x+b y+c=0$.

The first process of this test deals with circular obstacles. The distance between an arbitrary path segment candidate and the center of an arbitrary circular obstacle, $C\left(x_{c}, y_{c}, r_{c}\right)$, is calculated as below.

$$
d=\frac{a x_{c}+b y_{c}+c}{\sqrt{a^{2}+b^{2}}}
$$

It is obvious that the path segment candidate does not pass through the circular obstacle in $d \geq r_{c}$ case. Two intersection points of $a x+b y+c=0$ and $C\left(x_{c}, y_{c}, r_{c}\right)$, $\left(x_{i_{L C}}, y_{i_{L C}}\right)$, are calculated when $d<r_{c}$ in order to figure out the feasibility of the segment.

$$
\begin{aligned}
& x_{i_{L C}}=\frac{f_{i_{L C}} \pm \sqrt{f_{i_{L C}}^{2}-h_{i_{L C}} g_{i_{L C}}}}{h_{i_{L C}}} \\
& y_{i_{L C}}=-\frac{a}{b} x_{i_{L C}}-\frac{c}{b}
\end{aligned}
$$

where

$$
\begin{aligned}
& f_{i_{L C}}=-\frac{a}{b}\left(\frac{c}{b}+y_{c}\right)+x_{c} \\
& g_{i_{L C}}=x_{c}^{2}+\left(\frac{c}{b}+y_{c}\right)^{2}-r_{c}^{2} \\
& h_{i_{L C}}=\left(\frac{a}{b}\right)^{2}+1
\end{aligned}
$$

The path segment candidate is excluded when any of two $x_{i_{L C}}$ satisfies $x_{1}<x_{i_{L C}}<x_{2}$ since this means that some part of it is located inside of $C\left(x_{c}, y_{c}, r_{c}\right)$. This process is applied to all the possible combinations of a path segment candidate and a circular obstacle.

The second process, which is applied to the path segment candidates not excluded in the first process, handles polygon obstacles. In the case when some part of a path segment candidate is laid inside of a polygon obstacle, it should intersect with one or more sides of this polygon. The intersection point of an straight line, which arbitrary path segment is included in, and a straight line passing through two vertexes, $\left(x_{P_{1}}, y_{P_{1}}\right)$ and $\left(x_{P_{2}}, y_{P_{2}}\right)$, is expressed as follows.

$$
\begin{aligned}
& x_{i_{L P}}=\frac{\left(x_{1} y_{2}-y_{1} x_{2}\right)\left(x_{P_{1}}-x_{P_{2}}\right)-\left(x_{1}-x_{2}\right)\left(x_{P_{1}} y_{P_{2}}-y_{P_{1}} x_{P_{2}}\right)}{\left(x_{1}-x_{2}\right)\left(y_{P_{1}}-y_{P_{2}}\right)-\left(y_{1}-y_{2}\right)\left(x_{P_{1}}-x_{P_{2}}\right)} \\
& y_{i_{L P}}=-\frac{a}{b} x_{i_{L P}}-\frac{c}{b}
\end{aligned}
$$

The path segment candidate is excluded when this intersection point is laid on it, $x_{1}<x_{i_{L P}}<x_{2}$, and the side of a polygon obstacle with those vertexes as the end points, $x_{P_{1}}<x_{i_{L P}}<x_{P_{2}}$, at the same time. This process is applied to all the arbitrary combinations of a path segment candidate and a side of polygon obstacle. The safe path segment candidates are obtained from the acceptability test.

The construction of path candidates is completed by connecting the end points of the safe path segment candidates, which are called as nodes in the later part of this paper, on obstacles. The nodes on polygon obstacles are obviously their vertexes. This implies that all the sides of every polygon obstacles should be included in the group of path segment candidates. The path segment candidates on circular obstacles are obtained as the circular arcs between two nearby nodes on the same circular obstacle. When a part of a circular obstacle is overlapped by another circular obstacle, the path segment candidates on it and containing the overlapped part of its contour should be excluded. Two circular obstacles, $C\left(x_{c_{1}}, y_{c_{1}}, r_{c_{1}}\right)$ and $C\left(x_{c_{2}}, y_{c_{2}}, r_{c_{2}}\right)$, are defined to be overlapped when the following condition is satisfied.

$$
\sqrt{\left(x_{c_{1}}-x_{c_{2}}\right)^{2}+\left(y_{c_{1}}-y_{c_{2}}\right)^{2}}<\left(r_{c_{1}}+r_{c_{2}}\right)
$$

The intersection points of those two circles, $\left(x_{i_{C C}}, y_{i_{C C}}\right)$, are obtained as below.

$$
\begin{aligned}
& x_{i_{C C}}=\frac{f_{i_{C C}} \pm \sqrt{f_{i_{C C}}^{2}-h_{i_{C C}} g_{i_{C C}}}}{h_{i_{C C}}} \\
& y_{i_{C C}}=-\frac{a_{i_{C C}}}{b_{i_{C C}}} x_{i_{C C}}-\frac{c_{i_{C C}}}{b_{i_{C C}}}
\end{aligned}
$$

where

$$
\begin{aligned}
& a_{i_{C C}}=-2\left(x_{c_{1}}-x_{c_{2}}\right) \\
& b_{i_{C C}}=-2\left(y_{c_{1}}-y_{c_{2}}\right) \\
& c_{i_{C C}}=x_{c_{1}}^{2}-x_{c_{2}}^{2}+y_{c_{1}}^{2}-y_{c_{2}}^{2}-r_{c_{1}}^{2}+r_{c_{2}}^{2} \\
& f_{i_{C C}}=x_{c_{1}}-\frac{a_{i_{c_{C C}}}}{b_{i_{C C}}}\left(\frac{c_{i_{C C}}}{b_{i_{C C}}}+y_{c_{1}}\right) \\
& g_{i_{C C}}=x_{c_{1}}^{2}+\left(\frac{c_{i_{C C}}}{b_{i_{C C}}}+y_{c_{1}}\right)^{2}-r_{c_{1}}^{2} \\
& h_{i_{C C}}=1+\left(\frac{a_{i_{C C}}}{b_{i_{C C}}}\right)^{2}
\end{aligned}
$$

Two circular arcs on $C\left(x_{c_{1}}, y_{c_{1}}, r_{c_{1}}\right)$, called ' $\operatorname{arc} \mathrm{A}^{\prime}$ and 'arc B' connecting two intersection points given in (20) exist; one of them is located inside of $C\left(x_{c_{2}}, y_{c_{2}}, r_{c_{2}}\right)$, and the other one is not. The overlapped arc is figured out from the center of arc $\mathrm{A},\left(x_{A R C_{A}}, y_{A R C_{A}}\right)$, and the center of $\operatorname{arc} \mathrm{B},\left(x_{A R C_{B}}, y_{A R C_{B}}\right)$. 


$$
\begin{aligned}
& x_{A R C_{A}}=x_{c_{1}}+r_{c_{1}} \frac{\lambda_{x}}{\sqrt{\lambda_{x}^{2}+\lambda_{y}^{2}}} \\
& y_{A R C_{A}}=y_{c_{1}}+r_{c_{1}} \frac{\lambda_{y}}{\sqrt{\lambda_{x}^{2}+\lambda_{y}^{2}}} \\
& x_{A R C_{B}}=x_{c_{1}}-r_{c_{1}} \frac{\lambda_{y}}{\sqrt{\lambda_{x}^{2}+\lambda_{y}^{2}}} \\
& y_{A R C_{B}}=y_{c_{1}}-r_{c_{1}} \frac{\lambda_{y}}{\sqrt{\lambda_{x}^{2}+\lambda_{y}^{2}}}
\end{aligned}
$$

where

$$
\begin{gathered}
\lambda_{x}=\frac{x_{c_{1}}-\frac{a_{i_{C C}}}{b_{i_{C C}}}\left(\frac{c_{i_{C C}}}{b_{i_{C C}}}+y_{c_{1}}\right)}{1+\left(\frac{a_{i_{C C}}}{b_{i_{C C}}}\right)^{2}}-x_{c_{1}} \\
\lambda_{y}=-\frac{\frac{a_{i_{C C}}}{b_{i_{C C}}}\left(x_{c_{1}}-\frac{a_{i_{C C}}}{b_{i_{C C}}}\left(\frac{c_{i_{C C}}}{b_{i_{C C}}}+y_{c_{1}}\right)\right)}{1+\left(\frac{a_{i_{C C}}}{b_{i_{C C}}}\right)^{2}}-\frac{c_{i_{C C}}}{b_{i_{C C}}}-y_{c_{1}} \\
\text { If } \sqrt{\left(x_{A R C_{A}}-x_{c_{2}}\right)^{2}+\left(y_{A R C_{A}}-y_{c_{2}}\right)^{2}}<r_{c_{2}}, \text { the path }
\end{gathered}
$$
segment candidates containing 'arc $\mathrm{A}$ ' should be excluded. The ones containing 'arc B' are excluded when $\sqrt{\left(x_{A R C_{B}}-x_{c_{2}}\right)^{2}+\left(y_{A R C_{B}}-y_{c_{2}}\right)^{2}}<r_{c_{2}}$. The same process is needed to be applied to $C\left(x_{c_{2}}, y_{c_{2}}, r_{c_{2}}\right)$ for the verification of an unacceptable circular arc.

The optimal path from a landing site candidate to the original landing site is determined by applying Dijkstra's algorithm[14]. The visibility adjacency matrix is constructed from the lengths of the path segment candidates and the coordinates of the nodes.

The optimal path planning algorithm explained in this section is applied to all the landing site candidates obtained in Section 2. This provides the lengths of the path starting from all the landing site candidates. The one with the minimum path length is decided as the optimal landing site.

\section{Simulation}

Simulations are performed to verify the performance of the proposed landing site decision algorithm. The lunar lander is assumed to be placed stationary on the point $\left(x_{0}, y_{0}, z_{0}\right)$.

$$
\left[\begin{array}{l}
x_{0} \\
y_{0} \\
h_{0}
\end{array}\right]=\left[\begin{array}{c}
0 \\
0 \\
20
\end{array}\right](\mathrm{km})
$$

The original landing site on the lunar surface $\left(x_{O L}, y_{O L}\right)$ is defined as below.

$$
\left[\begin{array}{l}
x_{O L} \\
y_{O L}
\end{array}\right]=\left[\begin{array}{c}
-68 \\
50
\end{array}\right](\mathrm{km})
$$

The map of obstacles is given as shown in the following figure.

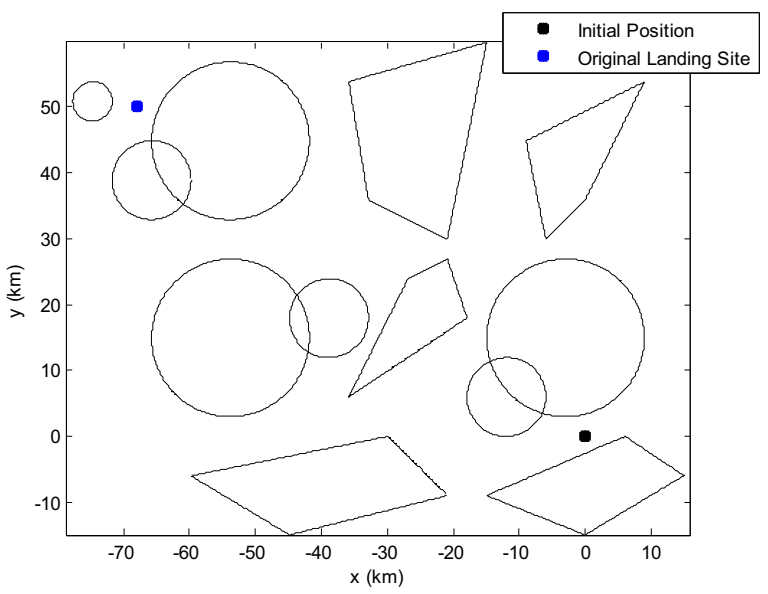

Figure 2. Coordinate System and State Variable Definitions.

\subsection{Landing Trajectory Optimization for Downrange Maximization}

The landing trajectory optimization is performed first to figure out the maximum downrange of the lunar lander. The initial conditions of the lunar lander are decided from (3) and (27).

$$
\begin{array}{llcc}
x\left(t_{0}\right)=0 & h\left(t_{0}\right)=20 \mathrm{~km} & V_{x}\left(t_{0}\right)=0 & V_{h}\left(t_{0}\right)=0 \\
m\left(t_{0}\right)=400 \mathrm{~kg} & \theta\left(t_{0}\right)=90^{\circ} & q\left(t_{0}\right)=0
\end{array}
$$

The terminal constraints are given in (4). The limits of attitude angle and its rate are defined as below.

$$
\begin{array}{ll}
\theta_{\text {min }}=70^{\circ} & \theta_{\text {max }}=110^{\circ} \\
q_{\text {min }}=-10^{\circ} / \mathrm{sec} & q_{\text {max }}=10^{\circ} / \mathrm{sec}
\end{array}
$$

The control inputs are given as follows.

$$
\begin{array}{ll}
T_{D_{\min }}=0 \mathrm{kN} & T_{D_{\max }}=1 \mathrm{kN} \\
T_{a t t_{\min }}=-50 \mathrm{~N} & T_{a t t_{\min }}=50 \mathrm{~N}
\end{array}
$$

The maximum and the minimum mass of the lunar lander are specified to decide the amount of available fuel during landing.

$$
m_{\min }=100 \mathrm{~kg} \quad m_{\max }=400 \mathrm{~kg}
$$

The moment of inertia and moment arm are also chosen as,

$$
I_{z z}=150 \mathrm{~kg} \cdot \mathrm{m}^{2} \quad L_{T}=1.0 \mathrm{~m}
$$

The optimal landing trajectory is obtained with GPOPS as follows. 


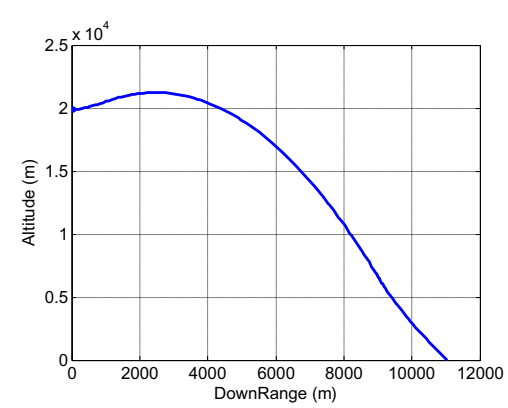

(a) Lunar Lander Trajectory

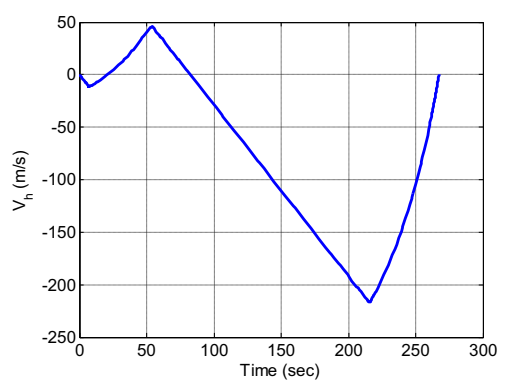

(d) Velocity Component in Altitude Direction

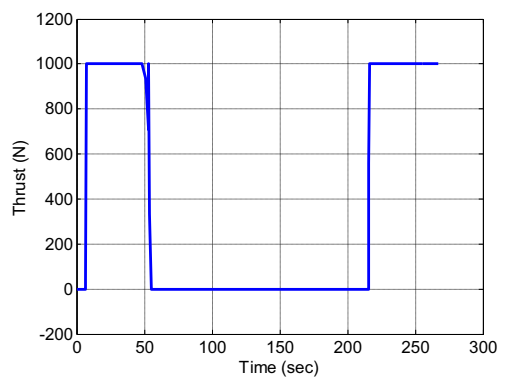

(g) Main Thrust

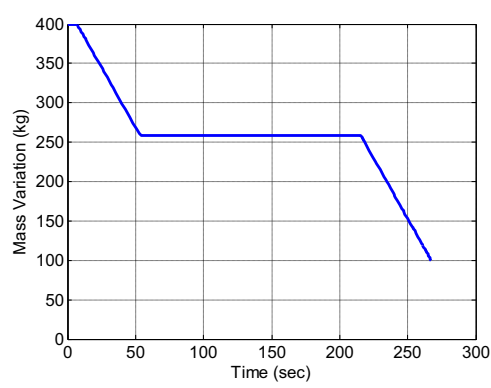

(b) Mass

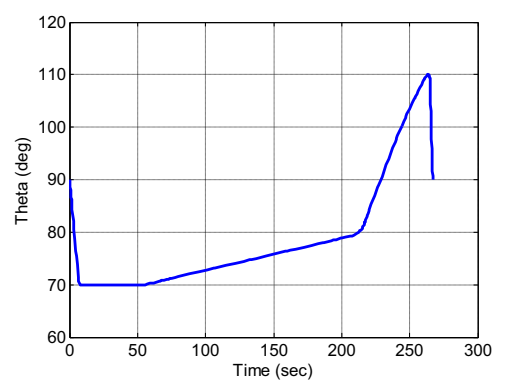

(e) Attitude Angle

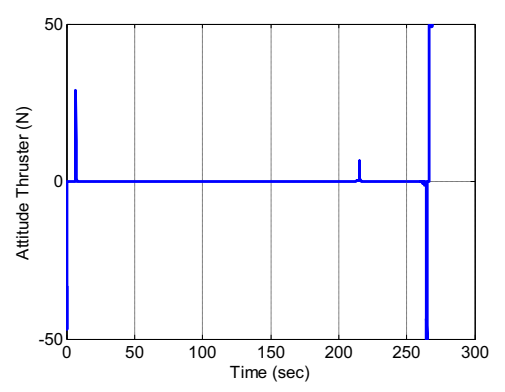

(h) Attitude Angle Rate Command

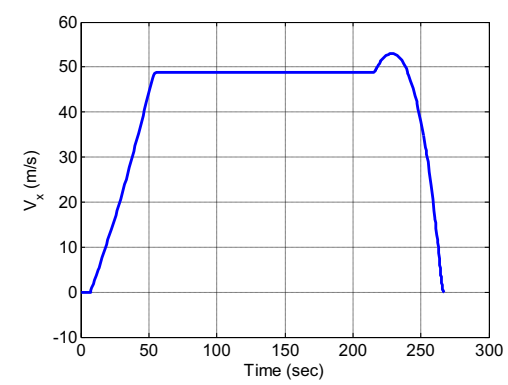

(c) Velocity Component in Downrange Direction

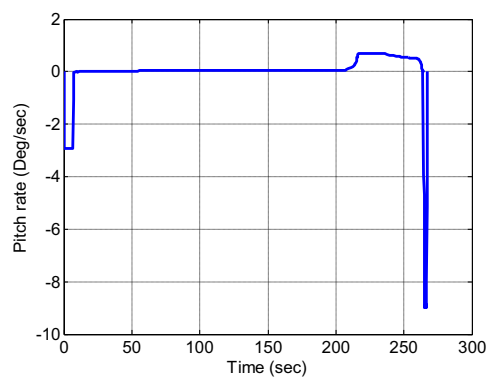

(f) Attitude Angle Rate

Figure 3. Landing Trajectory Optimization for Maximum Downrange.

The maximum downrange, $x_{\max }$, is $11.01 \mathrm{~km}$. As shown in Fig. 3-(c) to (f), state variables satisfy the terminal constraints for soft landing. The control inputs, the attitude angle and its rate are bounded by their own limits as given in Fig. 3-(e) to (h). All of the available fuel is consumed as addressed in Fig. 3-(b).

\subsection{Optimal Landing Site Decision with Path Planning Algorithm}

The outer-most contour is defined as the circle centered at the initial position on the lunar surface, $\left(x_{0}, y_{0}\right)$, with the radius of $x_{\max }$ figured out in the previous step. 120 points on this contour, dividing it in equal intervals, are chosen as the landing site candidates. The locations and shapes of obstacles are given as drawn in Fig. 2. The result of optimal landing site decision algorithm is obtained as Fig. 4.

Fig. 4 shows that the path segment candidates and nodes are generated from the given obstacle map. The cyan points, laid on the outer-most contour of the reachable landing region, are the feasible candidates of new landing site, and the red point located on $(-10.9949,-0.5762)$ is the optimal landing site. The green dotted lines are the feasible path segment candidates obtained with the optimal landing site, and the red circles on the obstacles are nodes. The solid magenta line represents the optimal path and its length is $92.4417 \mathrm{~km}$.

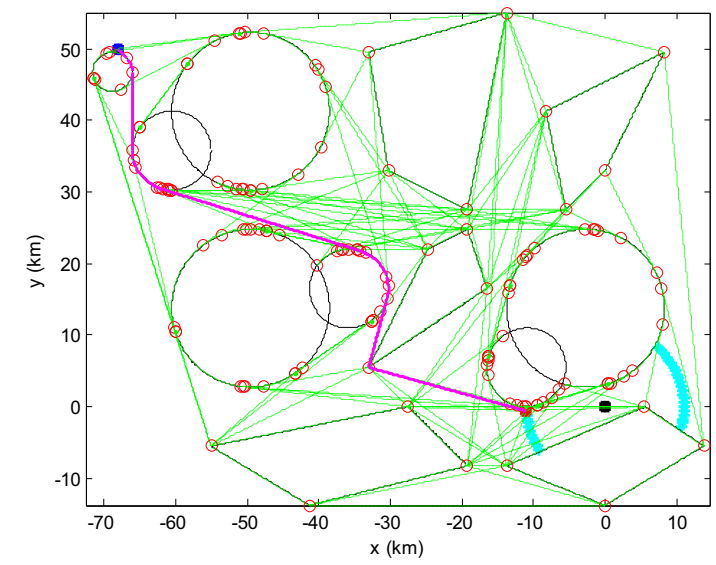

Figure 4. Optimal Path and Landing Site for Lunar Rover. 


\section{Conclusion}

The landing site decision algorithm for lunar lander is proposed in this paper. The outer-most contour of safe reachable region is defined by solving landing trajectory optimization problem. This problem is defined to figure out the maximum downrange with the terminal constraints and the energy limits in consideration. GPOPS is utilized to figure out the solution. The algorithm to find the shortest path between a landing site candidate and an original landing site is designed to select the most suitable landing site for lunar rover energy consumption minimization. The path segment candidates and nodes are generated from a given obstacle map by applying the theories of geometry. The shortest path is derived by applying Dijkstra's algorithm.

\section{Acknowledgment}

This work was supported by the National Research Foundation of Korea(NRF) Grant funded by the Korean Government(MSIP)(No. 2014M1A3A3A03034589).

\section{References}

1. A. R. Klumpp, Automatica, 10, 2, (1974)

2. R. R. Sostaric, J. R. Rea, AIAA Guidance, Navigation, and Control Conference, (2005)

3. F. Najson and K. D. Mease, AIAA Guidance, Navigation, and Control Conference, (2005)

4. B. Açıkmeşe, S. R. Ploen, Journal of Guidance, Control, and Dynamics, 30, 5, (2007)

5. L. Blackmore, B. Açıkmeşe, D. P. Scharf, Journal of Guidance, Control, and Dynamics, 33, 4, (2010)

6. B. Siciliano, L. Sciavicco, L. Villani, G. Oriolo, Robotics: Modeling, Planning and Control, (Springer Science \& Business Media, 2009)

7. V. J. Lumelsky, A. A. Stepanov, IEEE Transactions on Automatic Control, AC-31, 11, (1986)

8. V. J. Lumelsky, A. A. Stepanov, Algorithmica, 2, 14, (1987)

9. K. Fuzimura, H. Samet, IEEE Transactions on Robotics and Automation, 5, 1, (1989)

10. E. Gat, M. G. Slack, D. P. Miller, R. J. Firsby, IEEE International Conference on Robotics and Automation, (1990)

11. M. Gerke, Proceedings of the American Control Conference, 4, (1999)

12. N. A. Melchior, R. Simmons, IEEE International Conference on Robotics and Automation, (2007)

13. M. Tarokh, Fuzzy Sets and Systems, 159, 21, (2008)

14. E. W. Dijkstra, Numerische Mathematik, 1, 1, (1959)

15. B. J. Jeon, B. G. Park, M. J. Tahk, 2013 Asia-Pacific International Symposium on Aerospace Technology, (2013)

16. F. Junhua, C. Pingyuan, C. Hutao, Systems and Control in Aeronautics and Astronautics (ISSCAA), 2010 3rd International Symposium on, (2010).

17. K. S. Kim, G. H. Moon, J. W. Kim, J. W. Jeong, H. L. Choi, H. C. Shim, M. J. Tahk, The 2nd
International Conference on Robot Intelligence Technology and Application, (2013)

18. D. S. Kim, K. Yu, Y. Cho, D. Kim, C. Yap, Computational Science and Its Applications-ICCSA 2004, (2004)

19. G. H. Moon, Master's Thesis, Korea Advanced Institute of Science and Technology, Daejeon, Republic of Korea (2014) 